Meta

Journal des traducteurs

Translators' Journal

\title{
Bureautique et télé-bureautique en traduction
}

\section{Caroline de Schaetzen}

Volume 40, numéro 4, décembre 1995

URI : https://id.erudit.org/iderudit/004040ar

DOI : https://doi.org/10.7202/004040ar

Aller au sommaire du numéro

Éditeur(s)

Les Presses de l'Université de Montréal

ISSN

0026-0452 (imprimé)

1492-1421 (numérique)

Découvrir la revue

Citer cet article

de Schaetzen, C. (1995). Bureautique et télé-bureautique en traduction. Meta, 40(4), 682-693. https://doi.org/10.7202/004040ar

\section{Résumé de l'article}

L'auteur présente différents logiciels de bureautique destinés aux bureaux et aux service de traduction. Elle touche ainsi à de nombreuses utilisations de la bureautique : la gestion administrative des traductions, les divers problèmes relatifs au texte (clavier, format, etc.). la bureautique orale el écrite, la télébureautique orale et écrite ou l'archivage des documents d'utilisation que vous pouvez consulter en ligne.

https://apropos.erudit.org/fr/usagers/politique-dutilisation/ 


\section{BUREAUTIQUe ET TELE-BUREAUTIQUE EN TRADUCTION}

\section{Résumé}

L'antewr présente differents logiciels de burcantique destines anx bureaus ef anx somices de traduction. Elle pouche ainsi d de nombresese's urilisasions de la burcauthque : la gersfion administrative des traductions, les divers prohlemes

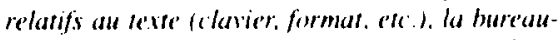

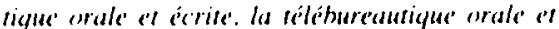

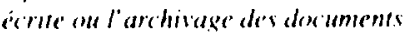

\begin{abstract}
The author discusses different office automatas sofinare insended for translation offices and deparments. She deals with many usses of office aufomathem translation manage ment. various technical prohlems lkevbeard, for mal. efe.1. oral and written office auromation. oral and written office communication systems. and doc cument archiveng
\end{abstract}

\section{DÉFINITIONS}

La bureautique désigne l'assistance aux travaux de bureau procurec par les techniques d'informatique et de télécommunication. l'organisation administrative, bref, toute la logistique du bureau et de son environnement. La bureatique implique donc des techniques informatiques mais aussi des techniques dorganisation classigues comme l'agencement des procédures, l'aménagement des structures, la reconversion des méthodes de travail, la formation du personnel, lergonomic des postes de travail, la seciologie et la piychologic du travitil. etc."

Sa fonction est de garder trace de ce qui se pasise : savoir ce qui est arrivé dans le passé el le retrouver en temps utile, d'une part. et d'autre part. fairc des plans et affecter des ressources (la première fonclion $n$ 'ayant de sens que pour permettre la secondes.

Linformatisation des bureaux est justifiée par des imperatifs economiques evidents: les coûts de main-d aruve sont de plus en plus cleves, en raison de l'inflation du volume al'informations traités et de la complexification croissante des procedures administratives: ce sont donc les bureaux quapres les ateliers les chefis d'entreprises désirent réorganiser. La traduction et la terminographie sont des secteurs de la burcautique parce quelles sont des elapes du traitement des dexuments.

\section{(GESTION ADMINISTRATIVE. DES TRADUCTIONS}

On peut appeler "bureautique de la traduction" la gestion de lattribution, de la circulation et de la progression des travaux de la traduction elle-même. Beaucoup de bureaux ou de départements de traduction ont programmé ou commandé la programmation d'une application de planification des travaux isf celui du Parlement, decrit dans les notes sur la traducfion automatique). Il n'existe cependant yue peu de logiciels commercialises. ETM ${ }^{2}$ est l un d'eux. destine aux bureaux el departements de Iraduction mais aussi aux traducteurs independants. Ecrit en CI.IPPER, I:TM tourne sous DOS, en reseau. Une version sous WINDOWS était en préparation a la fin de 109.3. P. Barber cite les ctapes de la traduction offre de services, devis, commande, traduclion proprement dite ; révision, correction, vérification des corrections, mise en forme, ajout de graphismes et de tableaux. "il y a licu. facturation. Lat gestion des traductions requiert, selon lui. les fichers ci-apres: clients, fournisseurs, historique da ajoh..

les efosts requis par une gestion des traductions sont quant ì eux : Etiquetles d'adresses, historique du "job", bon d'achat, liste de numéros de téléphone. Etat davancement des Iravaux en cours (par date d'échésuce, client, langue, fournosecur, numéro d'ordre des jobs. numeros de fournisseurs ou do clents), liste de clients. liste de fournisscurs. listes de langues et de specialites. statistiques.

las fithes des rficonts comportent le nom de la société, un numéro d'identificatıon, l'adresse du siège (nom du hàtiment, ruc, ville, pays, canton postal, numero de teléphone, de fax, de messageric) el celle de la facturation, qui peut en differer favec. pour la facturation, les instructions speciales el taux préférenticls éventuels). l.e nom du contact, avec son titre, son poste dans la scxiéte ou le ministere, son numero de telephone. sit fonction fourrer, traducteur, réviseur, photocomposeur. ctc.l, kur adresse, numéros 
de téléphone, de messagerie électronique el de fax individuels. s'il y a lieu. Une zone-note permet d'inserer des informations informelles (nombre d'exemplaires requis pour les travaux, instructions particulières pour la présentation, police).

Les fiches du job component le nom du contact. un numéro d'identification du job, la description du job (langues, instructions particulières, comme la remise du tapuscrit a un agent local du client pour approbation preliminaire avant photocomposition), la dale de remise lavec le degré d'impérativité et éventuellement le moyen d'y parvenir : envoi par courrier prive. par avion. etc.). les références du bon de commande, la date à laquelle le job a effectivement été remis. Les fiches de táches sont des fiches relalives à une partie du job : un ou plusieurs documents du job, une ou plusieurs langues dans lesquelles tout le document doit être traduit ou autres modes de division du travail (recherche doxumentaire, revision).

Les fiches des fournisseurs comporte quant a elles le nom de leur siciété ou bureau, leur titre, nom el prénom, leur poste, leur numéro d'identification, leur fonction (courrier, traducteur, réviseur, photocomposeur, etc.). leur adresse. numéros de téléphone, de fax, de messagerie électronique et de modem, leur equipement. notamment pur les traducteurs (PC ou nom, taille de disquettes, logiciel avec le numero de version. le type d'imprimante). leur langue maternelle, leur langue d'arrive habituelle, leur niveau de compétence (code-chiffre), leurs spécialites, leur tarif (ventilé par type de travail, paire de langues, domaine, degré d'urgence) et la base de calcul de la facturation (ligne, mot, rôle, du texte en langue source ou langue d'arrivece. par exemple, pour les traducteurs). date de dernière mise a jour (sans cela, certains traducteurs monteraient... très souvent leurs prix).

\section{EN MARGE DU TRAITEMENT DES TEXTES TRADUITS}

\section{Alphabets et signes dlacritiques}

Les micro-ordinateurs utilisent le code ASCII ( American Standard Code for Information Interchange"), basé sur un ensemble de caracteres utilisé par les fabricants de machines a ecrire électroniques americains. Les fabricants de microordinateurs ont travaille avec un second jeu de 128 caractères, non standardisé mais souvent aligné sur celui de Macintosh, d'IBM ou d'EPSON (longtemps leader dans la commercialisation des imprimantes). Deux organismes développent actuellement un nouveau jeu qui incorporerait autant de signes d'alphabers différents que possible. C'est l'ISO qui a commencé l'élaboration d'une norme, appelée 10646 . Elte a trouvé un pendant dans un projet de code. $\mathrm{Ce}$ dernier, appelé UNICODE, a été élaboré par les fabricants d'ordinateurs inotamment IBM. Apple, Microsoft. Xerox et Sun). En 1992, I'ISO décida d'incorporer UNICODE dans son projet de norme.
Comment, alors, rendre multilingues un clavier et un ecran d'ordinateur? Plusieurs solutions, boiteuses pour la plupart, ont été imaginces par les fabricants :

- Certains claviers, comme ceux du Grand-Duché du Luxembourg ou de la Suisse francophone permettent la frappe des caracteres francais et allemands. moyennant la perte de certains signes peu utilises ou le recours à la touche ALT.

- Des fabricants ont recemment construit des machines ou des logiciels intégres aux machines pour permettre de passer d'un clavier grec à un clavier français. Cela peul exiger la relance a chaud ("rebootage") du système ou le recours a une touche à chaque passage du clavier.

- Des sociétés ont cré des logiciels, surtout pour DOS jusqu'a présent, qui permettent de creer ou de modifier un jeu de caractères pour l'écran. $\mathrm{Ce}$ jeu peut ensuite être charge dans une carte graphique EGA ou VGA, comme EGA-FONT de MICROQUE. Cette firme a également programmé BILPOP, une interface disponible sur appel. BILPOP permet de contrôler les pilotes d'impri. mante installes, de choisir parmi six configurations de clavier différentes ou encore dentrer un symbole directement a partir du jeu de caracteres apparaissant a l'écran.

- Une série de logiciels de traitement de texte multilingues ont été mis, sans succes, sur le marché. les utilisateurs leur préférant les grands standards comme WORDPERFECT, WORD ou AMIPRO

- Des polices dans d'autres alphabets que l'alphabet latin sont disponibles pour toutes les applications et imprimantes tournant sous WINDOWS (a la différence de DOS, c'est WINDOWS qui gere l'impression et non plus les logiciels toumant sous lui). C'est le cas de WINDEAST pour les langues d'Europe de l'Est. Ou de KEY FOR WINDOWS. qui permet la frappe simultané en caracteres latins, cyrilliques el grecs. Le probleme est que le tri de ces caractères, cyrilliques, n'est souvent pas possible avec ces polices d'ecrans et d'imprimantes. Ni non plus la vérification orthographicogrammaticale des texles écrits avec eux.

\section{Lecteur de textes sténographies}

Sculs des traducteurs et des interprètes seniors ecrivent leurs traductions en sténo. Les interprètes formés dans les instituts pour traducteurs ont appris la technique de prise de notes des interprètes professionnels, buste sur l'idec. Quant aux traducteurs, wit ils dictent leurs traductions soit ils les dactylographient directement sur traitement de texte. Ils pourraient cependant ecrire a la fois en traitement de texte et dans une variété de langage sténographique. pour gagner du temps. AGILIWRITING ${ }^{3}$ est un logiciel de transcription automatique en anglais standard de textes dactylographies en langage sténographiyute AGILI. Cette expansion se produit au fur el a mesure de la frappe dans le langage stenographique ACilLI. 
Le dictionnaire d'AGILIWRITING contient environ $\varnothing(\mathbf{O O})$ abréviations et peut être complété à volonté par l'utilisateur. AGIl.JWRITING produit des decuments en sténo dans le traitement de texte de l'utilisateur et les restitue en «clair» sur demande.

AGILl est un systeme phonétique d'abréviation de l'anglais qui n'utilise que des lettres standard de l'alphahet anglais. En voici quelques principes. Ier lettres doubles sont simplifices f "adl" au lieu de "add") : les voyelles liees sont remplacees par "y" ou "w" ( aimmedrate" devient «imdy 1 ") : le son "in" long est remplace par "y" ("light" devient "lyt"): les voyelles el les consonnes muettes sont supprimeses. Ainsi, "Wrplenfmu th wwlbabl 1 dive the eypm urgr athendv nxwk" sera convert en "We are pleased to inform you that we will be able to deliver the equipment you require at the end of next week".

(e logiciel, dont on souhatteratit un equivalent pour la langue française, est eqalement utile aux journalistes, aux médecins, et bien sür aux secrétaires.

\section{Convertisseurs de formats et de polices}

les autorites puhliques. les Editeurs. les grandes tirmes, les bureaux de traduction. ont un probleme commun : l'échange de donnés électroniques avec des partenaires commerciaux, des clients ou de collegues. Les systemes d'explotation comme SYSTEME 7 de Macintosh ou WINDOWS de Microsoft sont tout d'atord incompatibles, même au sein du monde des PC: Ensuike, la multitude des applications tournant sur chacune des plates-formes qu'ils constituent l'est egalement. Jintin. mème si les récipiendaires disposent des mêmes applications que ceux qui leur ont foum less donnéces, il reste une autre divergence : les polices des imprimantes el de leceran (si l'utilisateur n'a pas les mêmes que l'auteur. la mise en forme est mutilisable). Jusqu'il y a peu. l'impression des documents était la seule manière de resoudre ces problemes, par exemple pour les traducteurs de publicatioms: ils fournissaicnt souvent une version sur papier prêle a clacher de leur traduction. Il sagit de pages pourvues de la mise en page, de la typographic et de la compositoon, y compros dans les dimenvions et avec les numéros de page définitifs du livere. selon les indications fournies par l'éditeur. Certains traducteurs indépendants travaillant pour plusieurs clients ou mème pour un seul bureau de traduction se voyaient également contraints d'apprendre plusieurs marques, et. pour une mêtme marque, plusieurs versions de tratiement de texte pour fournir leur traduction dans un format lisible directement par leur client. Les traitements de texte disposent d'un convertisseur. Dans leurs dernieres versions, ce convertisseur de formats peut fonctionner automatiquement; il peut donc. lors de l'nuverture d'un document uexolique", délecter le format d"origine du kexte, donc le type, sa marque et sa version du logiciel sur lequel al a été écrit puis convertir ce format dans sal mise en forme a lui. (e) convertisseurs ne sont pas parfiats, tant ien faut : ils perdent certaines fonctions de présentation de loriginal.

L.a demière solution, qui est la plus élégante. est un logiciel appelé ACROBAT, commercialisé par ADOBI: ll permel dechanger des documents complexes, entierement mis en forme, quel que soit te systeme d'exploitation. l'ordinateur, indépendamment des polices, des images, de l'application ou des applications originelles. Pour ce faire, ACROBAT convertit les donnesen orignelles en un format universel, appelé PDF: “Portable Dexument Format". Ce fichter peut êre crese de deux manteres. Si le dexument original est crés avece un logiciel qui peut ecrire des hichiers en POSTSC RIPT (un langage de description de contenu el de mise en forme de pages compris d'une seric de photexomponeuses et d'imprimanten a laser), un tel ficher peut etre converti en fichice Plof une "impression sur disque" (udisk printing") effectuec par un soun-programme d'ACROBAT, ACROBAT DISTILIIER. Pendant la conversion, l'utilisateur peut choinir la methede de compression du texte, des graphismes el des images et reduire kes pages en minialures pour laftichate. Autre mode de conversion en fichier PDP: : lutilisation d'un gestionnaire d'imprimante (un adriverm), le PDF WRITIER pour l'ecrilure sur disque du fichier. ACROBAT permet de visualiser un fichier PIPF: à l'écran ou sur imprimante. A l'ecran. la navigation s'effectue par des clies sur des boutoms, des fooms el des recherches de mots ou dittems. Des copies el impressions de portions de lexte sont egalement possibles. Des tables de matieres peuvent êre creses. I es fichiers PIDF sont de vériables documents électroniques, dans lesquels le lecteur ou lauteur peut créer des liens entre passages de textes, graphismes, images, des annolations mullt-auteur, yui implifient les relectures san reconversion. lin cas d absence de correspondance entre polices, ACROBAT prévient toute alteration de lat mise en page par unc substitution des polices Equivalentes.

\section{Decuments-types}

La collection REDACSOFT propose des mexlèles de formulatres et actes juridiques prêts à lemploi. II se compore de trois volumes : droit des seciétés, droit du travail et transmission dentreprises. Au wotal. 1 1.50) formulaires et acter juradiques, directement utilisable dam un tratement de texte sous WINIOOWS. Lechoix de l'anicle ou de l'acte seffectue an traver de sommaires successifs guidant liutilisalteur. Ensuite. 1) suffit de le copier dams son traitement de texte. Un boulon de lat barre d"outik de REISAC SOFT permet de basculer dams le traitement de texte thoisi, évitant le "coper-coller" de WINIOWW la harre d'outils reste active dans la fenetre proncipale du tratement de texte : l'utilisateur peut aims revenir à tout moment dans REDACSOFT pour choisir un autre modele. l.a collection couvre de manière exhaustive les domaines operationnels de leentreprise. Pour créer une SARL. par exemple, l utfit de chower le chapitre sur la constitution et de remplor un a un les modeles de 
lettres proposés. Le volume intitulé «Transmission d'entreprises" s'intéresse aux textes relatifs à la fusion d'entreprises, a la cession de documents de contrôle ou aux operations sur les baux commerciaux el les fonds de commerce ( 200 modèles).

Les traductions de courrier sont également assistees. COURRIER PARFAIT. commercialisé par DIDACTA (Bruxelles), est un programme résidan sous DOS. II permet de consulter une base de données reprenant l'ensemble de la correspondance commerciale française. aux segments desquels un index et une table des matières bilingues donnent un accès aise. On peut ensuite transférer automatiquement dans son traitement de texte tous les fragments ou lettres requis. Le programme symétrique SUPERBRIEVEN offre les memes fonctions pour le courrier commercial en neerlandais. SUPERBRIEFEN pour l'allemand et SUPERMAIL pour l'anglais.

On souhaiterait la multiplication de bibliotheques de ce type. Elles éliminent la frappe repetitive de traductions de textes juridiques mieux encore que ne peuvent le faire des bibliotheques de paragraphes.

\section{BUREAUTIQUE ORALE“ \\ Doublage de films}

Le doublage de films se fait sur une bande sonore séparte de celle des bruilages et de la musique. Les deux bandes sont ensuite mixces a l'issue de l'enregistrement de la voix des acteurs doublant en langue d'arrivée. Ce travail s'effectue dans des studios equipés de matériel specialiste. Les acteurs parlent dans un micro, devant un grand ecran de projection. Sur cet écran apparaissent, outre les images du film a doubler, un code d'identification des images (indiquant l'heure, la minute, la seconde et le numero du plan) et, en bas de l'ecran, une bande. Sur cette bande, appelée "rythmo", apparaît le texte que devra lire l'acteur. Ce dernier devra lire ce texte en parfaite synchronisation temporelle et parfois visuelle (imitation des mouvements des lèvres pour les images en gros plan). Le texte avance de droite a gauche sur l'ecran, au rythme de la progression du film. L'acteur, qui decouvre le texte au moment de sa lecture, doit terminer sa lecture au moment ou la fin de la ligne de texte disparait à gauche de l'ecran.

La grosse difficulté du doublage est la synchronisation labiale ou "lipsyncing" : au moment où l'acteur de l'ecran ferme ses levres pour dire «boow. par exemple. le texte en langue d'arrivec doit donc comporter une labiale. Il faut en outre que le debit du "doubleur m puisse s'accelérer ou ralentir au rythme de celui de l'acteur parlant en langue originale. Diverses techniques ont eté mises au point pour faciliter la solution de ces problèmes. Un programme appele WORDFIT, ulilisé en Grande-Bretagne, postsynchronise automatiquement les dialogues doubles avec l'image. Ce programme repere immedialement le début et la fin d'un dialogue sur la bande-image : il ajoute automatiquement de petites pauses ou abrege les sons en excès là où le requiert une synchronisation parfaite.

\section{Reconnaissance et synthise de la parole}

La parole est le moyen le plus direct d'echange d'informations utilisé par l'homme, qui réve dés lors de pouvoir s'adresser par ce médium à ces nouveaux collaborateurs que sont les ordinateurs. Lorsqu'il sera au point, l'ordinateur a entré vocale remplacera le dictaphone du traducteur. Quant a l'interprete. il pourra. sur un ordinateur capable de le comprendre a l'audition, consulter une banque ou un dictionnaire de termes pendant son interpretation. Enfin, la reconnaissance de la parole est l'étape préliminaire de l'interpritte automatique, dont plusieurs laboratoires ont déja sorti des prototypes...

Alors qu'on ne realise, en reconnaissance de la parole, que des dispositifs capables de reconnaitre quelques milliers de mots prononcés isolément par une seule personne. les recherches en synthèse de la parole en sont déjả a des raffinements comme le réalisme dans l'intonation. La synthese de la parole à partir du texte est donc pratiquement au point. La reconnaissance globale des mots isolés est au stade industriel. mais la reconnaissance analytique, celle de la parole continue. est toujours au stade de la recherche. La raison en est l'absence de silence entre les mots. qui pose un gros problème lors de la segmentation de la chaine parlee. La très grande variabilité que peut présenter un même discours selon la manière de parler (chant. murmure. voix enrouce su enrhumee) et le type de locuteur (enfant, femme. homme, vieillard, voix nasillarde, differences de timbre), sans parler des accents regionaux, complique également la reconnaissance. S'y ajoutent les perturbations qu'apportent le microphone (selon le type. la distance. l'orientation) et l'environnement (bruit. resonance). En outre. la parole est une combinaison d'informations de niveaux hétérogènes:

- phonéliques (la manière dont les sons influent les uns sur les autres dans un mot et une phrase):

- syntaxiques (structure de la phrase):

- sémantiques (sens du discours):

- pragmatiques (contexte dans lequel est prononcée la phrase).

Les chercheurs doivent donc faire appel a plusieurs disciplines: psychologie, linguistique, acoustique, informatique, theorie de l'information (traitement du signal). Ils doivent également traiter un grand nombre de données.

SPEACHSYSTEM a cré un système qui reconnaît la parole continue et la convertit en codes phonetiques el vice-versa. IBM a développé un systeme de reconnaissance vocale globale pour PC PS2 d'un vocabulaire de 200000 mots empruntés au vocabulaire des affaires. Les mots prononcés y apparaissent immédiatement à l'ecran: le système exige des pauses entre les mols et doit apprendre la voix de l'orateur. La KURZWEIL VOICE WRITER de 
KURZWEIL APPLIED INTELLIGENCE pouvait déja reconnaitre. en 1988, 15 (O) 10 mots (150 par minute) en parole continue avec une fiabilite de $95 \%$.

HEWLETT PACKARD a intégré la parole à la bureautique. Les messages écrits au HP Desk peuvent être annotés par des compléments vecaux. l.e créateur du dexument place des lcones dans son texte et y introduit des messages vocaux. L'utilisateur pointe sur l'icone pour l'entendre "parler". Tous les logiciels de traitement de texte qui integgreront ansi la parole sont intéressants pour les réviseurs de traduction et les experts, qui pourront ainsi corriger verbalement les travaux des traducteurs qui leur sont soumis. II l'est également pour les traducteurs consultant un expert a qui ils pourront exposer, dans la traduction ellemême, leurs difficuliés de traduction.

Le Japon a investi des milliards dans un télé phone interpréteur. La Régic anglaise des téléphones (British Telecom Research Laboratories) a fait de même. Le prototype anglais peut interpreter une conversation smple en anglais des affaires et ce. de l'anglaiss a l'espagnol, l'italien, le suédois et l'allemand. Chayue orateur dispose, apress entrainement du yystème a sa voix. d'un micro relie à un PC comportant le dictionnaire. Les ordinateurs sont programmés pour reconnaitre des mots-clés grâce auquel les machines repèrent les phrases. ce qui réduit la marge d'erreur dans l'analyse automatique des sons. le ystème reconnait les noms propres; il ne les traduit done pas et. lors de son interpretation. il en imite la prononciation originale par l'orateur. le choix des mots-clés est base sur un corpus de plus de centanes de milliers de phrases extraites de lettres datfaire ecrites en plusieurs langues. Des interpreles portatifs, de type "boitte noire" avec micro, on été mis au point pour les touristes. Il s'agit d'une sorte de Marabout Flash de langues parlés. (itons celui d'ADVANCED PROIDUCTS AND TECHINOL(OCIES de REDMONI). a Washington. (e traitement de texke oral portatif a piles est actives par commande vocale et émel des messages vocaux et ecrits sur un écran de 16 lignes. Des modules sur cartouches permetlent la traduction instantanee de phrases anglaises simples en françans. en allemand, en eupagnol et en italien.

In synthese. IBM a imagine un produit permettant l'acces des aveugles et des handicapes de la vue tant au PS/2 quiax programmes creses porur lui. SCREEN READER KFYYPAD permet d'entendre un texte affiche a leceran. L.utilisateur peut contrôler la vitesse et le mode de cette lecture synthétique (épellatuon lettre par leture. par mos. par ligne, par phrase. par paragraphe ou mode en comunu). (e logiciel a commande vexale permel de diriger l'affichage jusqu'en vingt cones de lescran et de faire lire a l'ordinateur les changements au fur et à mesure qu'ils ue produisent a l'scrian.

\section{TELÉBUREAUTIQUE ECRITE:}

\section{Internet}

Crés par lé gouvernement américain. INTERNET est un vaste réseau international, cooperatif. de réseaux interconnectés. Il permet à 3.2 millions d'ordinateurs de tous cypes el modeles. commandés par plus de $30(K)(X)(X)$ d'utilisateurs'. de partager des services et de communiquer direclement, comme s'il faisaient partic d'un ordinateur geant. global. Certaines organisations privees sponsorisent certain reseaux commerciaux, tandis que les gouvernements en financent d'autres à des fins de recherche et déducation. Liabsence de proprietaire complique encore ce mélange de composiantes publiques et privées. l.'ISOC. "Internet Sexiety» supervise la croissince du réseau et décide des normes qui lut sont applicables. Ser inembres sont des volontaires, recrutés parmi les utilisateurs. ISOC comporte encore un conseil compose de 8.5 societes, fondations et agences. Un groupe de membres invités siège en outre a l'" Intemet Architecture Board", responsable des normes promulguées par l'alnternet Ingineering Taskforce", un groupe auquel participent beaucoup de membres. qui échangent librement leurs idées et influencent la gestion du réseau. Chaque réseau attaché a INTERNET peut être dirigé par des gens qui édictent les règles de fonctionnement dudit réseálu. L'absence de prédominance d'un réseau a mis INTERNET, dirigé par ses utilisateurs, à labri de toute pression politique ou commerciale et lui confere son caractere eqalitaire communication de plusieurs a plusieurs et non de un à plusieurs. comme dans le jeumalisme traditionnel), cosperatif. $45 \%$ des utilisateurs appartiennent au monde éduca. thonnel (étudiants el facultés universitaires, éludiants et professeurs du secondaire, scientifiques) et $37 \%$ au secteur commercial.

Pour se connecter directement à Intemet et utiliser lous ses ervices (el pan uniquement la messageric par le truchement d'un réseau de messagerie de type COMP(ISERVE), il faut un modem et une connexion. par le truchement de BEIINET ou dTIINET BEI. Cill $\mathrm{M}^{\text {th }}$ L Les communications devant se faire en $\mathrm{TC} /$ IP ( "Transmission Control Protocol/ Internet Protocol"), une interface est requise, par exemple FTP Software's Packet Driver Specification (gratuite), une des plus confortables est celle qui ex fait par le truchement des librairies DL.I. de WINIOOWS I.utilisatteur de PC wou WINDOWS peut aussi acheter un interface pour INTIERNIET a peu de frais IAIR for WINDOWS. INTERNIET-IN-A-BOX, INTIERNET (CHAMEIIION) ou le recevoir comme "freeware" (WINSOCK PINC; AND FTP. MOSAIC FOR MICROSOFT WINIX)WSI le nombre de logiciels sous WINDOWS pourvus d'un protecole TC / IP es croissant. Mais, vu l'importance d'INTER NET, la connectivite au protocole TC / IP sera incluse a la prochane version de WINIOWS lui-même. De grandes entreprises font en outre migrer leur reseau vers TCP / IP. qui tend a supplanter la nome (OSI. Le's coùts sont particulièrement bas car ils n'incluent que la connexion au 
foumisseur d'informations et le cout de la ligne tćléphonique (l'accès à des ordinateurs, l'envoi de courrier. le transfert de fichiers, etc. sont gratuits. Aucuns frais ne sont encourus pour la durée d'acces ni pour lendroit du réseau où l'on va). L'utilisation est donc déjà rentabilisce par le remplacement des coups de téléphone intemationaux par la messagerie INTERNET. Les services offerts par INTERNET comprennent :

- un service de messagerie ;

- INTERNET RELAY CHAT, comme une radio amateur (bruyante et embrouillé) :

- un protocole de iransfer de fichiers (FTP. «File Transfer Protocol 2 ) pour charger des programmes et des fichiers volumineux d'ordinateurs eloignés :

- TELNET. pour faire fonctionner ces ordinateurs éloignés depuis votre PC :

- ARCHIE, VERONICA, JUGHEAD et WAIS, des outils pour consulter les immenses bibliotheques d'informations stockées sur INTERNET :

- GOPHER, un canal de déplacement rapide d'un point a l'autre du réseau :

- le WORLD-WIDE WEB, un des systemes de navigation les plus avancés. qui organise son contenu par sujet :

- MOSAIC, une sorte de panneau de controle en ligne qui permet de se déplacer dans la toile ("web») en pointant et cliquant avec la souris. Certaines firmes l'utilisent en interne :

- INTERNET TALK RADIO, qui diffuse des enregistrements sonores :

- CUSEEME, un système de conférence vidé permettant a 8 utilisateurs de se voir et de s'entendre sur leur ecran d'ordinateur.

L'autoroute de l'information' est une structure physique et une technologie de réseau a large bande annoncée par MCI Communications. Les impulsions photoniques (et non plus électriques) sont envoyées sur des fibres optiques (non plus à travers des fils de cuivre) a 10 Gigabits par seconde, au lieu des 2,5 actuels. Après numérisation el compression, l'information de tous lypes est ensuite transmise sous forme de signaux radio captables directement ou via un satellite. La lumière permet de transmettre beaucoup plus d'informations que l'électricité el dans un choix de frequences beaucoup plus large. L'information est codée sous forme de frequences et d'amplitudes d'ondes électromagnétiques. (On peut donc envoyer à la fois differents types de signaux et par consequent également plusieurs services, sur une seule ligne. L'échange de donnees informatisees, de sons, d'images, de films, offrira de nouveaux services dans de nombreux secteurs (chaînes cablees de télévision interactive de qualité cinématographique avec choix de la langue pour la bande sonore, réservation de places de theâtre. pendant lequel le consommateur $\alpha s^{\prime}$ assied» sur un fautcuil virtuel pour «essayer» sa place, enseignement à distance et téle-travail. grâce à l'envoi en temps réel de graphiques, images etc. en tout point du globe, par exemple echange de renseignements entre médecins pendant une intervention chirurgicale). INTERNET est quant à lui un réseau logique. II restera sans doute operationnel lorsque l'autoroute de l'information sera implantée, sa longévite. de l'experience qui s'y est accumulée, de sa robustesse, de ses améliorations continuelies. Sa structure physique, hétérogène donc lente pour certaines parties et très rapide pour d'autres. sera bien sûr modifiée à ce moment-là. L'interface de type (iUI s'y géneralisera sans doute, aux dépens de l interface basee sur les caractères, plus difficile à maitriser par le novice.

Signalons, sur INTERNET, un immense dictionnaire d'informatique et d'acronymes d'informatique ll existe d'autres dictionnaires, qui $n$ 'ont pas encore cté répertoriés a l'intention des traducteurs et des interprètes (voilà une tâche utile pour les associations de traducteurs!).

Les traducteurs et terminographes devraient être intéressés par les "newsgroups" de USENET, des forums dont les utilisateurs lisent des discussions sur un ou plusieurs sujets specialisés. Comme il existe des unewsgroups" sur absolument toute question, des problèmes de terminologie peuvent être soumis à des specialistes inconnus dans le monde entier. l'expé. rience a montré que les reponses à des questions difficiles n'excèdent pas trois jours. Le fichier de $F A Q$ (*frequently-asked questions») qui existe pour lout "groups contient en outre peut-être certaines réponses. La lecture de ces nouvelles esi en outre une source precieuse dinformations el de formation a des domaines. notamment de pointe. Ils sont organisés hiérarchiquement. Il existe des annuaires pour lout ce que peut offrir INTERNET. notamment ches MicroApplicationx. Les listes de publipostage cibles devraient également leur être précieuses, car ce sont des sources de spécialistes internationaux. Sur INTERNET, tout utilisateur peut en effet envoyer du courrier électronique a n'importe qui dont il connait l'adresse électronique. Mais il est en outre possible d'obtenir des listes d'adresses professionnelles, si on utilise INTERNET uniquement pour son service de messagerie. Ces personnes ne lisent pas de "newsgroups mais reçoivent les lettres par messagerie et elles peuvent en envoyer, afin de participer elles aussi aux discussions. Selon leur activité. les utilisateurs peuvent recevoir jusqu'à 7.5 messages par jour (qui ne sont donc pas tarifess).

\section{Videntex?}

Le videotex esi un système d informations structurtes en pages alphanumériques $\mathrm{et} /$ ou graphiques que I'on peut faire afficher sur un terminal, un ecran de PC ou un poste de télévision. Une page remplit un écran : elle est stockete en mémoire et peut donc être rappelee n'importe quand. Flle est composée ligne par ligne (et compone des indications de mise en page). $\grave{A}$ une des extrémiles du service se trouve le centre serveur, qui propose a ses utilisateurs les pages d'informations. A l'autre extrémité, les terminaux de 
consultation. Minitels ou micros pourvus d'un logiciel d'interface permettant l'émulation de Minitels, peuvent entrer en communication avec l'ordinateur-serveur par le réseau DCS cou TRANSPAC en France) par l'intermédiaire de leur modem. Le terminal doit disposer d'un deconteur adapté et les informations reçues sont affichées sur ecran apres décodage. le terminal peut envoyer lui-même des donnees, que le serveur stockera en mémoire el auquel il répondra. le branchement sur TRANSPAC est effectué directement par le Minitel: Il ne reste des lors a l'utili. saleur qu"à composer le numero d"appel du serveur et a sélectionner le service yu il souhaile.

Une norme uniformise en France la présentation et la transmission des données ainsi que les procé dures et messages necessaires a la transmission. D'où la standardisation et la facilité de Télétel, qui permet lacces à tous les serveurs sans apprentissage de protocole, comme c'est le cas pour les serveurs qui ne sont pas sur Minitel. D'où aussi son manque de souplesse (cascade de menus). Une interface, ou, pour les gros serveurs, un ordinateur frontal, $c^{\circ}$ est-a-dire un ordinaleur transformant les données vidéotex en données compatibles avec l'ordinateur et vice-versia. peuvent rendre un matériel infonnatique incompatible conforme a la norme vidéotex. Les Minitels peuvent mémoriser et composer automatiquement une série de numéros d'appel.

Le vidéctex permet un acces rapide a des services d'informations. notamment un annuaire des traducteurs travaillant dans 40) langues et quelque 125 domaines, affiliés a la SFI (Sixiété française de (raduction). Certains ervices sont gratusts, d'autres wont tarifés lau prix de la communication. légèrement majore) et d'autres entin. payants (soumis a contrat). le Minitel est. en France, devenu un standird de fait et comme tel, il est incontoumable. Ausst la plupart des systemes de traduction antomatique et des banques de terminologie s'y branchent-ils: ce'est le cas AEURODICAUTOM. de NORMATERM et du systeme de iraduction SYSTRAN. L.e CILF y diffuse

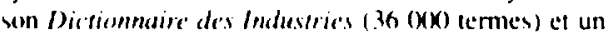
dictionnaire de nélogismes (sur base des ficher de la Banque des Mots). La Belgique a adopté, sans grand succés jusqu'a présent, la norme Téstel en mars 198x. In bottin des traducteurs el une base de données sur les organismes s'excupant de langues (services ministernels, écoles de langues, etc.) ont été intégrés à som Minitel.

\section{Télécopie}

La télécopie est la reproduction à distance de toul document écrit. dactylographié ou photographié. Le dixument est analysé par un télécopieur, appareil qui effectue un balayage par faisceau lumineux d unt page décomposée en lignes. Il engendre ensuite un signal lumineux converti en donnees binaires. (hez le correspondant, un autre télécopieur reconstitue le document original.

Le télecopieur peut être relié à un réseau loxal, ou a un réscau public (notamment aux satellıtes).
Couplés à un modem. des logiciels permettent également les télécopies sur micro-ordinateurs. Il existe de nombreux moxlèles de copieurs de qualités diverses et à prıx réduit. dont une série combinent téléphone. repondeur, télecopieur et photocopieur. C'est le nombre de lignes balayées au millimètre qui détermine la qualité des copieurs. Ils sont de plus en plus utilisés pour transmettre des traductions, notamment urgentes ou courtes, des devis et des questions urgentes de tenmınologie. La télécopie est donc devenue une concurrente redoutable de la poste (une page A4 est (ransmise n'impone où en quelques secondes). d'autant plus que les équipements sont en outre de plus en plus compacts.

Quels sont les aplus» d'un bon produit-fax pour ordinateur 11 :

- un modem VI7, qui permet la transmission a $144($ M) bits/s :

- une télécopie-couleurs, possible uniquement entre des equipements du même fournisseur, pour le moment :

- l'annotation des copies entrantes:

- une bibliotheque de pages de garde ou une interface ave un traitement de texte qui possede des modeles de pages de garde de fax :

le support des normes TR 29 CL.ASS I el ('LASS 2 el de CAS

- l'émission d'une télécopie comportant plusicurs documents :

- la visualisation d'unc télecopic sur écran. avant son envor: :

- la compatibilité avec plusieurs types de scanners ou le support de l'interface TWAIN pour scanners:

- des répertorres lélephoniques partageables avec d'autres applications:

un modem de formal "cante de crédit" pour les ordinateurs portables.

\section{Télé-écriture}

Pratiquement détrônée par la télécopie. lat télé écriture est la transmission de dessins, schémas. graphiques ou textes manuscrits, au moment même sù ils se font. Le dispesitif se compense d'une tablette reliée a un stylo spécial et à un ordinateur. lui-mème relié à un téléviscur el a une ligne téléphonique par medern. I.es informations graphiques sont tracees sur un papeer posé sur la tahletle. L ourdinateur traduit en donnees hinaires les mouvements du stylo et les affiches sur lescratl de l'émetleur en même temps qu'il les transmet a l'ordinateur du destinataire, qui décokle a son tour ces informations. Is procédé de codage est tres simple et nécessite peu d'informations a transmettre. Un debit faible est suffisint, ce qui permet de combiner la télé-écriture avece la conversation téléphonique. La tablette est équipé de touches de fonction permettant un choix de couleurs. I'effacencent de liscran. la designation deun point de lécran ou la mémorisation de son contenu. Comme la télé copte, lat téte-écriture permet l'envoi de traductions 
manuscrites. Assis à cốté de l'orateur en correspondance telephonique, l'interprète de liaison peut surtout, gráce à elle, copier sur une tablette de téléecriture ce qu'écrit ou dessine l'orateur. tout en interprétant au téléphone le discours de son client. La télé-écriture peut appuyer les conversations techniques sur des termes : les expens peuvent illustrer leurs explications téléphoniques de diagrammes el de schémas divers.

\section{Messagerie électronique}

La messagerie électronique est la communication de textes informels. messages courts le plus souvent. transmis sans souci de présentation. Ces messages remplacent une série de lettres, de déplacements dans et a l'extérieur d'une entreprise ou d'une administration et de coups de téléphone. Ils présentent l'avantage de ne pas déranger le correspondant el de l'alteindre (des enquêtes ont montre que $70 \%$ des appels (éléphoniques $n$ aboutissent pas). Les logiciels de messagerie électronique disposent de procédures de securité pour harrer l'accès aux personnes qui n'y sont pas abonnés (mot de passe affiche et modifiable sur demande de l'utilisateur). Avec la messagerie, le correspondant prend connaissance des messages quand il le désire. par consultation de sa boîte aux lettres. Ou bien, il reçoit les messages après un délai déterminé, par remise directe. Ou encore. il les reçoit automatiquement dès qu ii est libre. Les identificateurs logiques (mot de passe, nom du destinataire) permettent d'envoyer et de recevoir des messages en tous lieux car ils remplacent les identificateurs physiques (numéro de téléphone. de photocopie) L'utilisateur peut sauvegarder le contenu des messages. le copier, l'imprimer ou acheminer le message ailleurs. Les messages sont de plusieurs types :

- personnel (pour un seul destinataire):

- privé (à ne pas montrer à sa secrélaire, par exemple):

- assoni d'un accuse de reception (accuse automa liquement envoyé a l'émetteur dès que le recepteur a pris connaissance du message).

Un message indique au destinataire la presence de courtier dans sa boite, dans les systèmes à temps partage. el parfois. l'arrivee de messages y est signalée en cours du travail. Les supports de la messagerie sont variés: télécopieur, téléphone, vidéotex mais surtout ordinateur, notamment via le réseasu de télécommunication INTERNET (voir ci-dessous)

La messagerie électronique est très répanduc. Grâce à elle. les commandes peuvent être adressées 24 heures sur 24 aux traducteurs. Les traductions de messages urgents peuvent lui être expediés n'importe quand lorsque le destinataire ne les comprend pas. De plus. le traducteur peut recevoir les commandes destinces a des collègues en vacances. qui ont demandé leur déroutage ches le traducteur «de corveen. Ce demier y répond à leur plince ou les renvoie chez d'autres traducteurs. Des services de messagerie électronique sont en outre inclus à des harkques de terminologie (TERMIUM).

\section{Télétraduction 11}

Les développements de télébureautique écrite changent la profession du traducteur et de l'interprète. Le télétravail recouvre plusieurs situations

- le travail électronique à domicile, consistan a travailler a domicile et non dans les lieux de travail habituels. en utilisant les technologies de l'information ef des communications pour réaliser sa tâche et rester en contact avec une ou plusicurs entreprises:

- le centre-satellite, une unité relativement autonome a l'intérieur d'une entreprise. séparéc physiquement de la maison mere, qui reste en contact constant avec elle par télécommunication :

- le télelocal, qui réunit des equipements électroniques partagés par différents utilisateurs, lesquels peuvent étre des employes de diverses sociétés ou appartenir a une societé trop petite pour faire l'acquisition de ces structures. Il peut également s'agir de travailleurs indépendants

- le telétravail nomade, travail mobile ou itinerant : travail de professionnels qui, par les télécommunications, peuvent rester en contact avec les entreprises utilisant leurs services. quel que soit le lieu où ils se trouvent:

- le teleservice : services ou produits informatıonnels dispensés par le réseau des télécommunicattons (téléformations, services de reservations)

Les traducteurs, surnout indépendants mais a présent également salariés, ont eté parmı les premiers a s'adonner au travail électronique a domicile. La Chemical Company, en Allemagne. fait appel it des traducteurs indépendants travaillant à la demande. en liaison avec l'entreprise pour les traductions pharmaceutiques. Le Bonner Übersetzungsbüro est un bureau de traduction auquel les traducteurs sous-traitants sont connectés en télélocal. Il envisage la tréation d'un service d'interpretation simultané de conversations. téléphoniques. Le Centre à distance de télétraduction Voltaire de la Société Loracom empioiera des traducteurs qui travailleront a distance des entreprises clientes ou a domicile. en liaison avec le Centre. Les traducteurs pourraient également proposer leurs activités en téléservice. comme les terminographes. d'ailleurs (par exemple sur INTERNET). Les interprètes peuvent être considérés conme des télétra vailleurs nomades. lorsqu'ils ve connectent aux bases de donnees terminologiques de leurs clients ou aux programmes de gestion administrative de leurs emplsyeurs éventuels.

\section{TÉLEBUREAUTIQUE ORALE 12}

\section{Téléconférence assistee par ordinateur}

La téléconférence assistée par ordinateur est une conférence par le biais de la messagerie électronique. 
une conférence étalée dans le temps et l'espace. Elle permet de dialoguer par écrit avec un ou plusieurs collègues ou confrères. de prendre connaissance de leur avis et d'intervenir pour communiquer soi-même des informations, générales ou destinées à un participant en particulier. La communication avec te service de messagerie peut être interrompue n'importe quand. Les messages envoyés en l'ahsence du destinataire sont enregistrés. Les avantages de la téléconférence sont nombreux: l'économie des frais de voyage et de séjour. le temps, pour les participants. de lire des informations à leur rythme et d'y réfléchir, d'interrompre momentanement le dialogue pour se consacerer a un travail urgent ou pour prendre des informations complémentaires avant de répondre à une question. etc. Aussi. cest par ce canal que les experts internationaux se sont concertés bors de l'alccident nucléaire de Tree Mile Island aux F́tats-I/nis, Ies interprètes de conférence trouveraicnt plus de débouches grâce à cette infrastructure, qui ferait economiser leurs frais de mission aux organisateurs des conférences.

\section{Audioconférence}

Laudioconférence ou téléconférence audiographique se fail dans un sludio d'enregistrement. L'equipement du studio comprend des micros, des diffuseurs de sons, un dispositif d'identification de chaque intervenant. Des dispositifs de téle-ecriture el de télécopic completent le service. La voie de transmission utilisé es motamment TRANSPAC en France. avec des lignes téléphomiques à moyen débit. Les principales villes françaises disposent d'un studio d'audioconference. qui peul être loué par les entreprises (télécentre). les PTT équipent en outre sur demande les entreprises de studios prives (location ou vente). Less réunions de négeciation, demandant un engagement emotionnel (donc l'examen des visages) des participants, se prêtem mal a ce type de confé rence, au contraire des réunions de travail. Pour la tarification, seule la durée est prise en comple : la distance ne l'est pas : le coût devrait donc enfin haisser atec l'avènement de l'autoroute électronique.

\section{Visioconférence}

À l'audicxonférence, la visioconférence ajoute la transmission de l'image. Les studios. Equipes de cameras et d'ecrans recepteurs, mettent en liaison deux groupes de quelques personnes. Lat transmission de l'image nécessite des circuits à large débit. U/me camera est installé devant chaque participant loorateur est délecté par une régic automatique. yu transmet alors son image aux autres participants, landis que lui-méme voit le visige du demier lexuteur. Une vue panoramique donne en outre, sur un mur, une vae densemble du studor des correspendants.

lat multiconfirence connecte plusieurs salles en lecation.

\section{Vidéotransmission}

L a vidétransmission permet de réunir plusieurs centaines de personnes dans des salles ade cinéma" équipés d'un écran géant. Munies d'un micro, ces personnes voient le conférencier, filmé par une caméra vidéo, et peuvent lui parler. Kissinger a utilisé ce mode de conférence a l'un de ses retours d'URSS pour répondre en même temps, en EUROVISION. aux questions de tous les grands journalistes européens. Les journalistes étaient installés dans un des studios publics de vidéotransmission de leur pays respectif.

La téléconférence supprimera un grand nombre de conférences et décentralisera sans doute certaines prises de décision (on pourra appeler un cadre en pleine réunion dans le studio dentreprise).

less différents types de téléconférencés décrits cidessus modifieront les conditions de travail de l'interprète. Celui-ci ne se déplacera plus, pour travailler, que dans les locaux de son client ou des sludios. Les interprètes pourraient se voir appeles plus souvent a prester dans kes réunions de travail. Ieur profession sen trouvera banalisée et salns doute moins bien rétribuée. ce que l'élargissement du marché de l'interpretation devrant compenser. L'interprete pourra. en técconférence, étaler ses prestations dans le temps et consulter sa decumentation entre deux interventions. Face a ces économies, les conversations de couloir et autres rencontres en marge des colloxues, le plaisir du dépaysement des conférenciers et des participants aux collexques ne peseront plus lourd.

\section{ARC:HIVAl;F:}

Pour l'archivage dans les bureaux. ce qui so passera demain peut déjà être observé dans certaines bibliothèques, transformées en centres d'informations. (ertaines entreprises utilisent elles aussi les techniques moxernes de stoxkige d'informations : (ieneral Motors équipe ses concessionnaires en vidécodisques, pour leur faire utiliser la nomenclature (iM pour leurs commandes de pieces dktachées. En outre, des sociétes francaises de cervices meltent des documents sur microfilms et microfiches, a partir d'une reproduction photographique.

Pourquoi de nouvelles méthodes d'archivage". Ie papier submerge les bureaux: il esl lourd. peu maniable, peu accessible, encombrant. polluant, consommateur de matières premières el d'énergic (la futurologie nous prédit un achoc papier» aussi violent que le cho petrolier). Einsunte, les techniques classiques d'archivage consistent en une suite d'operations consommant du temps, de la main-d'aruvre, de l'argent. de la place. Voici une echelle des capacites des supperts de stockage. (In disque rigide de "mainframe" (gros ordinateur) comporte 70 Gigabyes. II peut dono stocker l'écuivalent de

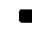

- envirun I 750 bihles de 40 Méga ou 40 (x) (XX) de caracteres:

- environ 2 8(M) annuaires téléphoniques de $25 \mathrm{Méga}$ ou 25 (X)o (x) de caractères:

- environ $140(x)(0)$ romans de $5(X) \mathrm{K}$ ou $5(X)(X)()$ caracteres, qui prendraient 4.2 kilometres de rayonnage dans leur version sur papier ${ }^{\text {? }}$. 
Pour la traduction et l'interprétation, comme pour toutes les professions de bureau, l'archivage est indispensable. L'administration du traducteur exige un ordre minimal dans les textes traduits, les sources bibliographiques et orales (experts, confretes), documentaires et terminologiques, le stockage des glossaires.

\section{Archivage micrographique}

La micrographie est l'ensemble des techniques et methodes utilisées pour transcrire. reproduire et exploiter des documents sur un support micropholographique (pholographie a échelle réduite) par microfilmage. Les microformes designent l'ensemble de ces supports microphotographiques : microfilms. microfiches. etc. Pour être exploitec, une microforme doit être visualisée a l'aide d'un projecteur special (lecteur). Les images peuvent être filmés par caméra mais les microformes peuvent aussi être produites directement à partir de handes magnetiques par l'ordinateur sur des imprimantes COM. Certains matériels utilisent des microprocesseurs pour la recherche automatique. $D$ autres permettent des mises à jour par adjonction de nouvelles vues et noircissement des vues perimees. La micro-edition est moins répandue en Europe qu'aux titats-Unis, l'obstacle ayant été l'absence de reconnaissance de ces documents par les tribunaux comme preuve.

\section{Videomation}

La vidéomation est un procédé d'archivage et de consultation de toute information ecrite, a partir d'un document original ou microfilmé par des moyens audiovisuels. Les equipements de vidéomation se composenı de postes de consultation à ecran. Ces postes reçoivent. par télévision en circuit fermé. l'image des doxuments désirés. Un système de télécommande à distance de lecture et d'ensembles émetteurs de ces documents (caméras TV + système de positionnement) est installe dans les lieux de classement. Les documents peuvent donc être visualisés directement, sans traitement ou codification du texte consulté, à partir de l'image du suppon original. Cela évite les déplacements de personnes ou de documents entre services. La visualisation se fait sans attendre et globalement, par page. Sans être déplacé. un document peut être consulté par plusieurs personnes a la fois, permettant une discussion a distance entre ces personnes. Une copie sur papier de l'écran de consultation est possible, pour letudier plus longuement. Les textes consultés peuvent être de tous types (plans, cheques, livres, photos...)

La vidécomation peut donc servir aussi bien pour la gestion documentaire que pour les gestions de dossiers de patients, de radiographies, les banques, etc. Généralisée, elle éviterait le déplacement des traducteurs de grosses administrations jusyu'au service documentaire. Ce serait le cas des rénnions préparatoires au renouvellement des conventions ACP.CEE. par exemple. Ces réunions se déroulent souvent dans des pays d'Afrique. des Caraibes ou du Pacifique, ou des equipes de traducteurs, disponibles 14 heures par jour, accompagnent la delegation d'interpretes pou les sessions de travail. La copie et la transmission immediates aux traducteurs des documents demandés par les participants au cours de la séance amélioreraient la qualité des prestations des interprètes.

\section{Disque optique}

Également appelé *CD-ROM* ou adisque compacto, le disque optique lit l'information selon la technique de lecture par laser. L'écriture sur ce support se fait par numerisation. Les copies sont impossibles. L'espace est de l'ordre des $7(X)$ Mega d'information pour les petits $\mathrm{CD}$. Certains disques sont réinscriptibles. Il existe déjà des serveurs de $\mathrm{CD}$ à peine plus grands que I'unité centrale d'un PS / 2, ce qui permet d'associer le CD et la télématique, ators qu'on imaginait, it y a quelques annees, que ces deux techniques seraient concurtentes. Le ( $D$ nécessite une cante d'interface (mais pas Windows) et un lecteur, interne ou exteme, de CD. Les logiciels de recherche des informations sont foumis avec le CD et sont souvent copier sur le disque rigide. S'ils sont résidents en mémoire vive, le CD peut être consulté sans sortir de l'application en cours (traitement de texte, notamment). meme s'ils tournent sous DOS, et des portions de la hase peuvent être copies dans cette application L'accroissement des CD-ROM s'accompagne de nouveautés technologiques qui rendent leur acces encore plus facile et contribuent donc a leur diffusion. Actuellement, la tendance va vers des CD-ROM conçus pour toumer sur des lecteurs reliés à des PC compatibles ou à des ordinateurs Macintosh. Ou bien. un disque est lisible sur n'importe quel lecteur ( $c^{\prime} \mathrm{es}$ le cas du CD-ROM Gevaen). Ou encore, le CD-ROM existe dans les deux versions. Mais prèn de $80 \%$ des disques sont pour les ordinateurs compantibles seulement. Autre tendance, des versions Windows pour $44 \%$

Le CD represente un progres important dans le traitement du langage naturel. En effet, les $P C$ actuels disposent de memoires de masse de 200 Méga, ce quı réduit fortement les possibilités de consommation massive de linguistique informatique. Des mémoires forement accrues supportent des applications beaucoup plus puissantes, aussi bien pour la correction orthographique ou stylistique que pour la traduction automatique. Mais le disque optique est d'abord un support idéal de stockage des données statiques it comme les dictionnaires. Aussi la plupart des editeurs de dictionnaires publient-ils actuellement une version sur disque compact de leurs ouvrages. Citons :

- Zyzomys : Dictionnaire de $49(00)$ noms communs. 20100 noms propres. 30000 synonymes et un atlas, avec plusieurs dizaines de cartes en couleur. Distribue par Hachelle.

- Base de données dictionnairiques de Brandstetter (Wiesbaden), comportant : Brandstetler (ompac's Dictionary of exact Science and Technology (ende-en. 115000 entrées).

- Esselte Studium Dictionary (sv-en-sv, $240($ (K) entres) ; Gyldendal Dicrionary (da-en-da, 110 (x) 
entrées): Kunnskapsforlaget Dicrionary (no-en-no, $\mathbf{5 5}$ (X)0 entrtes): NTC's American ldioms Dictiemary (8)(0) expressions idiomatiques): Sansyusya Dichonary of Science and Technology (en-de-ja. 175 (OK) entrées) : (Gendai Puhlishing's Dictionary

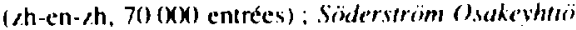
Dicnomary (fi-en-fi. $70(\mathrm{x})$ ) entrées): VolecrsNoordhoff Dictionary (en-nl-en. 753 pages) : Zasnichelli's II Nuero Ragazami (en-it-en, 128 ()(x) entrés): Zamichelli's Fire Language' Database' (en-fr-de-it-es): Harrap's Shorer Dictionary (fr-en-fr. 350 (KX) entrées): Harrap's Dicrionary of Data Processing ifr-en-fr. 18000 entress: Harrap's Schence Dictionars (fr-en-fr. 30000 entrées) : Harrap's (oncose Dictumary (de-en-de. $95(x)$ entrés): Harrap's Basiness Documary (en-fren. 487 pages).

- Oyford English Dicrionnary: 400000 mots el $18(x)(O) 0$ citations.

- PERINORM: $1100(0)$ nomes definies par les invituts hritanniques. altemands, français (BSI, DIN, AFNOR) et par I'ISO).

- TERMDOK : DOC comportant des extraits de TNC (6 000) concepts). la base de termes RTT (multilingue. $28(\mathrm{KK})$ concepts). TEPA (multilingue. $9(0)$ (concepts). les glossaires de TNC (multilingues. 28000 concepts). NORMATERM (en-fr. 47 oOO concepts), des extratis de TERMIUM l $/(0)(x)$ concepts).

- LEXITRON : dictionnaire néerlandais explicatif Van Dale ( 100 (MK) entrées).

- LEXICODISC : Bertelsman Lenicon (12 volumes).

- Meciraw-Hill : 100 (OK) lermes du Dichonary of Scicentific and Teronteal Terms et le concise' Encrefopaedia (1.5 volumes).

- Microsofi Bookshelf : 10) livres de reférence el dictionnaires encylopédiques anglais.

- Crolier: Encyclopédic de l'Académie Américaine De caractere universel et en anglais, elle est composé de 20 volumes et de 30 (000 articles. Distribuée par Hachette

- Graml Robers álestromique' : il comprend 800000 mots.

Liédition d'un (D)-ROM multimédia passe par plusieurs phases et requiert un PC 486 adapté au graphisme cécan a hate résolution. RAM de 16 méga. disque rigide alteignant la taille requise par le volume de l'application it developper pour le (D)).

- Scénario. F́tablissement de la succession des séquences, des choix arborescents proposés. du type d'illustrations utilisées. Lat question des dromts d'auleur doil êtré réglée

- Edition. Confection de chaque type de donnés utilisées dans le (D) (sons, images. films videos, textes) avec les "édlecurs" adéquats. II sagit a silvoir respectivement des logiciels de traitement de textc. pour le texte, de logiciels comme WACE EDIT, pour ke son. de VIDEO FOR WINIOOWS de Microsoft pour les films, de PHOTOSTYLER d'Aldus, pour les photos. Celte étape comprend la numérisation de l'image (le son CD audio est déjà numérisé et peut être utilisé tel quel).

- Montag'e. Assemblage des éléments el programmaton des éléments interactifs a l'aide d'un logiciel de développement. un logiciel ou un langage de programmation d'auteur comme MULTIMEDIA VIEWER de Microsoft. DIRECTOR de Micromedia. AUTHORWARE (pour l'enseignement, car il calcule le taux de réponses correctes donnés lor de l'utilisation du CD, au fil du cours multimedia. Il peut faire approfondir les notions incomprises de l'apprenant). ("es lui yui ser a l'auteur à "construire" la structure arborescente du CD. a échafauder l'interactivité. a programmer des "boutons" ou les rones d'écrans pour des dialogues, des explications. etc. Si le programme est surtout destine a la recherche d'informations, il faudra y ajouter un programme ad hoc (TEXTWARE ou un gestionnaire de bases de données comme ACCESS).

- Rohert af Collens. dictionnaire en-fo traductif des Éditeurs Robert et Collins, vendu ou non avec le lecteur portable de (D)-ROM Data Discman de Sony.

- Test, une phrase qui prend $30 \%$ du temps de la confection d'un CD. Il s'agit de la confection avec un CD Recordable d'une version test qu'il faudril ensuite modifier, déboguer. Notamment parce que le CD est plus lent qu une version de programme et de donnees sur disque rigide. L'appareil de création CD-R ("compacl disk recordable") de la matrice d'un $C D$ coûte environ $200(K)$ ) francs (APPLE, RICOH).

- Marrice. Confection du CD Recordable qui servira de matrice el sera dupliqué par une société spécialisee (SONY. PHIL.IPS. notamment mais aussi ORDA-B). L'impression d'un (D) a 1 (OOO exemplaires coûte environ 50 franç pièce.

Notons yue la (D)-I ou (DD-ROM interactif de Philips, utilise sur des postes de télévision. n'est pas adapté au monde professionnel mais aux produits destinés aux loisirs et aux enfants.

\section{Notes}

1. L.a bureautique Outils ef applacations. J.-P. de Blasis, les Editions d'Organisation, Paris, 1985.

2. Development of Management Package for Translators in Translation Management". Machine Thans/anion Todas: Translating and the Computer 15. P. Barber., ASI.IB, L.onden, 1993.

3. The Wrile Remedy for Wriker's Cramp Ac;llL WRITING;. AgiliTyping Lid. London. 1993.

4. "Outils de hureautique et de télématique pour la traductuon". Lehende Sprachen. C. de Schaetzen. langenscheidt. $n^{\prime \prime}, 1990$. 
5. The Internet. A Giohal Business Opportunity. Deb Cameron. Computer Technology Research Corp.. Charlesion. South Carolina. 1994.

6. Belgian Research Nerwork. 8. tue de la Science. 1040 Bruxelles. Monsieur Pierre Bruyere. EUNET BELGIUM, p/A KUL Leuven, Departement Computer Science. Celestijnenlaan. 200A. 300)1 Leuven. INTERNIC «Information Services Reference Desk". General Atomics, PO Box 85608. San Diego. CA $92186-9784$ peut indiquer tous les autres points d'acces à INTERNET, pour tous pays, toutes applications et tous types d'utilisateurs.

7. "L'avenir des communications à large bande en Europe». I\&T Magazine, Debora Mackenzie. Communication des Communautés europeennes. DGXIII, Luxembourg, 1994.

8. Ou bien: The Whole Internet User's Guide and Catalog, Ed Krol, O'Reilly, ou encore: The Internet: Compler Reference. Harley Hahn and Rick Stout. Osborne McGraw-Hill. Pour les débutants : Interner Guide for New'Users. Daniel Dem. McGraw-Hill.

9. "Outils de bureautique et de telématique pour la traductionm, op. citt.

10. Le micro communiquant. Fax-minitel-messagerie vocale ef écrite-groupw'are, A. Odier, M. Zennaki. Marabout, Alleur, 1994.

11. Le télétravail, R.-M. Lemesle et J.-C. Marot, PUF. coll. "Que sais-je "?m, Paris, 1994.

12. "Outils de hureautique el de télématique pour la traduction m, op. cit.

13. La plus grande bibliotheque du monde (Foyles, a Londres) occupe 7000 mètres carres de 48 kilometres de rayonnages, avec ses 5000000 de volumes.

14. En juin 1994, le CD-ROM Directory comptait déja 7000 titres.

CAROLINE DE SCHAETZEN Centre de Terminologie de Bruxelles. Institut Libre Marie Haps, Bruxelles. Belgique 